BULLETIN Bulletin hispanique

HispaniquE Université Michel de Montaigne Bordeaux

117-2 | 2015

Métamorphose(s) : représentations et réécritures

\title{
Le mythe du phénix dans le théâtre de Lope de Vega ou les métamorphoses du temps
}

\section{Alexandre Roquain}

\section{(2) OpenEdition}

\section{Journals}

Édition électronique

URL : http://journals.openedition.org/bulletinhispanique/4092

DOI : 10.4000/bulletinhispanique.4092

ISSN : 1775-3821

Éditeur

Presses universitaires de Bordeaux

Édition imprimée

Date de publication : 15 décembre 2015

Pagination : 657-674

ISBN : $979-10-300-0041-2$

ISSN : 0007-4640

Référence électronique

Alexandre Roquain, « Le mythe du phénix dans le théâtre de Lope de Vega ou les métamorphoses du temps », Bulletin hispanique [En ligne], 117-2 | 2015, mis en ligne le 15 décembre 2018, consulté le 23 avril 2019. URL : http://journals.openedition.org/bulletinhispanique/4092 ; DOI : 10.4000/ bulletinhispanique.4092 


\title{
Le mythe du phénix dans le théâtre de Lope de Vega ou les métamorphoses du temps
}

\author{
Alexandre Roquain \\ Université de Bourgogne
}

Cet article a pour objet d'analyser les fonctions du mythe du phénix (Les Métamorphoses, Ovide, Livre XV) dans le théâtre de Lope de Vega. À travers l'analyse littérale de quelques fragments de " comedias ", on se demandera si le phénix, au-delà du simple cliché, a une fonction temporelle et sert une poétique de la métamorphose du temps.

Mots-clés : Lope de Vega, phénix, mythe, Ovide, métamorphoses, temps.

Este artículo tiene por objeto analizar las funciones del mito del fénix (Las Metamorfosis, Ovidio, Libro XV) en el teatro de Lope de Vega. A través del análisis literal de algunos fragmentos de comedias, nos preguntaremos si el fénix, más allá del mero estereotipo, asume una función temporal y está al servicio de una poética de la metamorfosis del tiempo.

Palabras claves : Lope de Vega, fénix, mito, Ovidio, metamorfosis, tiempo.

This article aims at analysing the functions of the myth of the phoenix ("The Metamorphosis», Ovid, Book XV) in Lope de Vega's plays. Through literal analysis of some of his works, we will study whether the phoenix, beyond the simple cliché, has a temporal function and can serve a poetics of the metamorphosis of time.

Keywords : Lope de Vega, phoenix, myth, Ovid, metamorphoses, time.

\footnotetext{
A U SiÈcle D'OR, la mythologie a profondément influencé la littérature et 1 en particulier le théâtre. Tout lecteur assidu de comedias se souviendra des multiples allusions à Adonis, Vénus, Circé, Médée et à tant d'autres
} 
personnages. Ces références aux mythes antiques apparaissent la plupart du temps de façon épisodique au cours de l'œuvre théâtrale. Le développement des traductions des textes antiques au $\mathrm{XVI}^{\mathrm{e}}$ siècle a permis la redécouverte de grands textes fondateurs comme Les métamorphoses d'Ovide, œuvre traduite six fois durant ce même siècle. Lope de Vega avait sans nul doute lu ces ouvrages de Bustamante ${ }^{1}$, Viana ${ }^{2}$ ou Pérez de $\mathrm{Moya}^{3}$, mais il est fort probable qu'il connaissait la version originale des textes d'Ovide : l'Art d'aimer, les Amours ou Les métamorphoses. Lope de Vega ne se contente pas d'introduire dans ses pièces de simples allusions aux personnages ovidiens. En effet, sept comedias mythologiques (Adonis y Venus, El laberinto de Creta, La fábula de Perseo, El vellocino de oro, El marido más firme, La bella aurora, El amor enamorado) du dramaturge sont une réécriture des Métamorphoses comme l'a démontré Juan Antonio Martínez Berbel dans El mundo mitológico de Lope de Vega. Siete comedias mitológicas de inspiración ovidiana ${ }^{4}$.

Le mythe du phénix a inspiré poètes, dramaturges, historiens ou philosophes de tous temps. Prenons deux exemples. En 1630, José Pellicer de Tovar, célèbre pour ses prises de position contre la comedia nueva et ennemi juré de Lope, consacrait un ouvrage exclusivement au phénix : El fénix y su historia natural 5 . $\mathrm{Au} \mathrm{XX}^{\mathrm{e}}$ siècle, Gaston Bachelard s'intéressait vers la fin de sa vie à cette figure mythique dans Fragments d'une poétique du feu et analysait, dans le cadre d'une perspective phénoménologique, les images de cet oiseau merveilleux ou " oiseau de feu ${ }^{6}$ " éternel.

Surnommé de son vivant "Phénix des beaux esprits" (Fénix de los ingenios) en raison du caractère prolifique de son ouvre et de sa capacité extraordinaire à produire autant de chefs-d'œuvre, Lope de Vega - on le sait - était considéré comme un être hors du commun, un " monstre de la nature " selon Cervantès. Je ne m'attarderai pas, dans la présente étude, sur l'antonomase élogieuse Phénix-Lope car cet aspect a déjà été élucidé en détail par la critique, en

1. Voici la version des Métamorphoses de Bustamante au sujet du phénix : "En la tierra de Assiria ay vn aue que llaman Fenix, aquella aue, quando ha viuido quinientos años, haz vn nido de muchas especias, y metese en el: de la fuerça de la calentura de las especias enciendese el nido, y arde y quemase alli el Fenix: y quando es toda esta aue hecha ceniza, leuantase de alli luego vn gusano, que poco a poco se va tornando en la forma que era primero despues quando es aue entera, lleua el nido en el ayre, y dexale alla. " Jorge de Bustamante, Las transformaciones de Ovidio, Anvers, en casa de Pedro Bellero, 1595, p. 222 (édition d'origine).

2. Pedro Sánchez de Viana, Las transformaciones de Ovidio: traduzidas del verso latino, en tercetos, y octauas rimas, por el licenciado Viana. En lengua vulgar castellana, con el comento y explicación de las Fabulas: reduziendolas a Philosophia natural, y moral, y Astrologia, e Historia, dirigo por Hernando de Vega Cotes y Fonseca, Valladolid, 1589.

3. Juan Pérez de Moya, Philosophía secreta de la gentilidad, Madrid, éd. Carlos Clavería, Cátedra, «Letras Hispánicas », 1995.

4. Juan Antonio Martínez Berbel, El mundo mitológico de Lope de Vega. Siete comedias mitológicas de inspiración ovidiana, Madrid, Fundación Universitaria Española, 2003.

5. José Pellicer de Tovar, El fénix y su historia natural, a costa de Pedro Coello, mercader de libros, Madrid, Imprenta del Reyno, 1630.

6. Gaston Bachelard, Fragments d'une poétique du feu, Paris, Presses Universitaires de France, 1988. 
particulier par Aurora Egido qui consacre un article essentiel au mythe du phénix principalement dans la poésie de Lope (ses Rimas, La Filomena, La Circe, La Gatomaquia), en précisant que le phénix apparaît comme l'incarnation du poète dans son ouvre, et en soulignant la nécessité d'une recherche approfondie sur ce mythe dans la prose et le théâtre de Lope. Elle fait allusion notamment à une base de données informatique (Teatro español del Siglo de Oro ou TESO), apparue à la fin des années 1990, qui facilite le repérage des occurrences du terme phénix ${ }^{8}$. Aurora Egido signale enfin que le terme fénix apparaît plus de 1000 fois dans toutes les œuvres numérisées tous dramaturges confondus et 469 fois chez Lope. La récurrence du signifiant et, pourrait-on dire, l'omniprésence du mot dans la comedia lopesque légitime l'exploration de ce mythe à l'aide de cet outil. Mais bien avant de découvrir l'article d'Aurora Egido nous avions été sensibles à une présence notable de l'oiseau miraculeux dans les pièces de Lope de Vega. Comme Aurora Egido consacre l'essentiel de son article aux surnoms de Lope, nous ne prendrons pas en compte cet aspect dans notre étude.

Compte tenu de l'abondance des citations à notre disposition, il a fallu restreindre le champ d'exploration et ne considérer que les recréations littéraires du mythe du phénix sous l'angle des Métamorphoses d'Ovide. Nous avons opté pour un classement qui rende compte des différents usages du phénix dans ce corpus. Rosa Romojaro propose une série d'outils analytiques dans un ouvrageclé sur le mythe classique et distingue cinq fonctions ${ }^{9}$ :

fonction topique et érudite

fonction comparative

fonction d'exemplification

fonction re-créative ou métamythique

fonction burlesque

Nous nous appuierons sur un corpus de 50 comedias provenant de toutes les séries dramatiques afin de rendre représentatives les constantes observables en ce qui concerne la réécriture du mythe du phénix. L'objectif de la présente étude n'est pas d'extraire des statistiques ni d'attribuer un pourcentage à telle ou telle fonction. À partir de ce vaste corpus, nous choisirons d'analyser quelques comedias où l'empreinte du mythe est la plus visible. Il semble nécessaire de s'intéresser d'abord aux fonctions topique, érudite comparative et burlesque dans la mesure où de nombreuses citations incluant la mention fénix vont dans le sens, à première vue seulement, d'une instrumentalisation du mythe par Lope. Est-ce à dire que le recours à l'oiseau phénix n'est que pur cliché et n'obéit qu'à un système de codes prédéfinis et largement utilisés par les poètes

7. Aurora Egido, «La Fénix y el Fénix. En el nombre de Lope ", in Maria Grazia Profeti, "Otro Lope no ha de haber", Atti del convegno internazionale su Lope de Vega, Florence, Alinea, "Secoli d'oro ", 2000, vol. I, p. 11-49.

8. Il s’agit de Teatro español del Siglo de Oro (TESO), dirigé par M. del C. Simón Palmer, Madrid, Chadwick, Healey, 1998, C.D-Rom.

9. Rosa Romojaro, Las funciones del mito clásico en el Siglo de Oro, Barcelone, Anthropos Editoral, 1998. Cf. p. 37-41 au sujet du phénix. 
du Siècle d'Or ? Nous tenterons de répondre à cette question lorsque nous aborderons la fonction de réélaboration. Nous cherchons en effet à savoir, à travers l'analyse littérale de quelques fragments significatifs du corpus, si le phénix a une fonction temporelle dans la comedia lopesque.

Le phénix est un oiseau fabuleux qui renaît de ses cendres après s'être consumé sur un bûcher d'aromates. Le mythe est développé dans le quinzième livre des Métamorphoses d'Ovide aux vers 391-407 ${ }^{10}$. Contrairement à d'autres figures mythiques, Ovide ne consacre que seize vers à l'oiseau fabuleux. Il n'est pas inutile de les citer dans leur traduction française ${ }^{11}$ :

Cependant tous ces animaux doivent à d'autres les principes de leur existence ; mais il y a un oiseau, un seul, qui se renouvelle et se recrée lui-même ${ }^{12}$; les Assyriens l'appellent le phénix. Il ne vit ni de grains ni d'herbes, mais des larmes de l'encens et du suc de l'amome. À peine a-t-il accompli les cinq siècles assignés à son existence qu'aussitôt, posé sur les rameaux ou la cime oscillante d'un palmier, il construit un nid avec ses ongles et son bec pur de toute souillure. Là il amasse de la cannelle, des épis du nard odorant, des morceaux de cinname, de la myrrhe aux fauves reflets; il se couche au-dessus et termine sa vie au milieu des parfums. Alors du corps paternel renaît, diton, un petit phénix destiné à vivre le même nombre d'années. Quand l'âge lui a donné assez de force pour soutenir un fardeau, il décharge du poids de son nid les rameaux du grand arbre et il emporte pieusement son berceau, qui est aussi le tombeau de son père. Parvenu à travers les airs légers à la ville d'Hypérion, il le dépose devant la porte sacrée de son temple.

À notre connaissance, le plus long passage sur le phénix dans l'œuvre de Lope est celui que l'on trouve dans le Troisième Livre de El peregrino en su patria et qui se compose de 66 vers. Nous pouvons en citer quelques extraits significatifs ${ }^{13}$ :

10. Ovide fait également allusion à l'oiseau phénix dans la sixième élégie du deuxième livre des Amours.

11. Ovide, Les Métamorphoses, XI-XV, Paris, Les Belles Lettres, "collection des universités de France ", 1991, p. 133-134. Nous reproduisons ci-dessous l'original en latin :

Haec tamen ex aliis generis primordia ducunt;

una est, quae reparet seque ipsa reseminet, ales;

Assyrii phoenica uocant; non fruge neque herbis,

sed turis lacrimis et suco niuit amomi.

haec ubi quinque suae compleuit saecula uitae,

ilicis in ramis tremulaeque cacumine palmae

unguibus et puro nidum sibi construit ore,

quo simul ac casias et nardi lenis aristas

quassaque cum fulua substrauit cinnama murra,

se super inponit finitque in odoribus aeuum.

Inde ferunt, totidem qui uiuere debeat annos,

corpore de patrio paruum phoenica renasci.

Cum dedit huic aetas vires, onerique ferendo est,

ponderibus nidi ramos leuat arboris altae

fertque pius cunasque suas patriumque sepulcrum

perque leuis auras Hyperionis urbe potitus

12. C'est nous qui soulignons.

ante fores sacras Hyperionis aede reponit.

13. Lope de Vega, El peregrino en su patria, Madrid, éd. Juan Bautista Avalle-Arce, Castalia, "Clásicos Castalia », 1973, p. 274-276. 


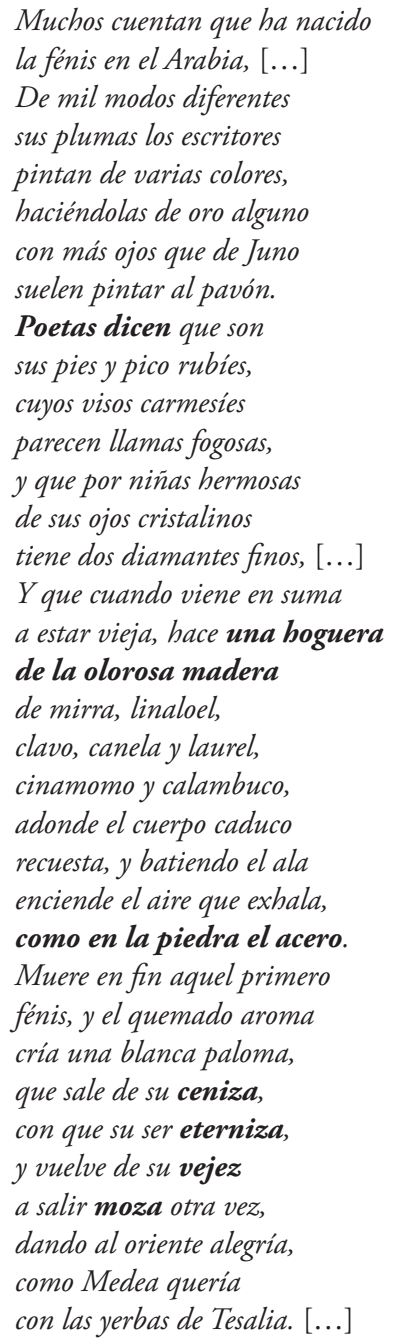

Bien que El peregrino ne fasse pas partie de notre objet d'étude, il constitue un témoignage fondamental au sujet de l'oiseau mythique. Cette longue citation est une réécriture des Métamorphoses comme le montre la description du bûcher odorant où les mots d'Ovide sont repris à la lettre par Lope : la cannelle, le cinname ou la myrrhe par exemple. De fait, on verra par la suite que Lope fait explicitement référence à Ovide dans son théâtre. Mais on observe qu'il signale, dans les quinze premiers vers cités, la richesse et la variété de la tradition littéraire relative au phénix, dont il se considère, autant que d'Ovide, l'héritier. Les pluriels et l'indéfini : Muchos cuentan, De mil modos diferentes, alguno, Poetas dicen que ... sont un hommage de Lope aux poètes qui l'ont précédé et qui ont contribué aux réélaborations successives du mythe. Lope a dû probablement s'inspirer en particulier de Lactance (Carmen de ave 
phonice $^{14}$ ) ou de Claudien ${ }^{15}$. Il ne nous appartient pas, dans les limites de cet article, de traiter ce problème de filiation. Ces vers du Peregrino offrent, par le seul jeu des rimes, une représentation condensée du mythe du phénix puisque le terme ceniza est lié à eterniza dans une poétique d'un temps cyclique où le vieillard retourne à son éternelle jeunesse. Notons que " moza» fait également écho à la rime $-i-z a$ et que les mots "vejez " et "otra vez ", qui lient la vieillesse au renouvellement temporel, participent également de l'allitération en $z$, précédée de celle en $s$ des mots "sale ", "su ", "ser ", "salir ", comme un phénomène sonore de la transformation. Ces sifflantes accompagnent non seulement l'envol de la colombe renaissante mais annoncent aussi l'apparition de la magicienne Médée. Ces allitérations peuvent rappeler aussi le bruit du battage du fer. En effet, Lope de Vega compare le processus d'auto-consumation du phénix à l'acier battu par la pierre. Cette fusion du métal semble être une recréation de Lope et l'on reviendra ultérieurement sur cette image. Le poète introduit un élément remarquable : la colombe, oiseau immaculé dont le symbolisme biblique ne peut être écarté. Il faut y voir sans doute l'empreinte d'un phénix se rapportant au divin. En effet, notre corpus contient des pièces hagiographiques et Lope y réserve à la figure mythique un traitement bien particulier. L'intrusion de Médée, qu'Ovide n'associe pas au phénix, semble lier le mythe de l' " oiseau de feu " à la magie noire.

Dans son ouvrage consacré aux pièces à caractère mythologique de Lope, Juan Antonio Martínez Berbel affirme que la mythologie a subi bien des adaptations au cours de l'histoire et en particulier au Siècle d'or ${ }^{16}$ :

El acceso a la mitología es menos directo que nunca en nuestro Siglo de Oro. A las alteraciones medievales hay que añadir el tamiz moralizador medieval, las lagunas en la tradición; y a todo esto hay que añadir el hecho importante de que cada obra supone un punto de vista que altera de nuevo el material mitológico. El resultado de todo este complejo proceso es una nueva mitología, cuyas fuentes inmediatas ni siquiera son las originales, sino a menudo, diccionarios, traducciones, mitografias que, como en el caso de Lope, esconden sutilmente el Ovidio original (en este caso) sustituyéndolo por un Ovidio renacentista al que, posteriormente, cada autor convertirá en un Ovidio personal.

C'est à cet Ovide personnel que nous allons nous intéresser dans les pages qui suivent. Avant d'envisager la fonction re-créative, nous pouvons citer quelques occurrences qui relèvent en apparence davantage du cliché que de la réélaboration.

14. Il carmen de ave phoenice di Lattanzio, Catane, éd. Emanuele Rapisarda, Centro di studi sull' antico cristianesimo, 1952.

15. Au sujet du phénix dans l'Antiquité et les premiers siècles du christianisme, cf. R. Van Den Broek, The myth of the phoenix, according to classical and early christian traditions, Leyde, E. J. Brill, 1972.

16. Juan Antonio Martínez Berbel, op. cit., p. 31. 


\title{
1. LE PHÉNIX : UN MOTIF POÉTIQUE RENOUVELÉ
}

Les nombreuses citations font apparaittre quelques usages systématiques. L'une des caractéristiques essentielles du phénix est son unicité et sa rareté comme en témoignent les premiers vers d'Ovide à ce sujet : " una est ... ales ${ }^{17}$ ". Lope de Vega s'appuie sur ce caractère unique de l'oiseau dès qu'il s'agit de mettre l'accent sur la grande valeur d'un objet et son caractère précieux comme on peut l'observer dans Peribáñez y el comendador de Ocaña ${ }^{18}$ :

COMENDADOR [...] Si sirviera una dama, hubiera dado

parte a mi secretario o mayordomo

$o$ a algunos gentilhombres de mi casa.

Estos hizieran joyas, y buscaran

cadenas de diamantes, brincos, perlas,

telas, rasos, damascos, terciopelos,

$y$ otras cosas extrañas y exquisitas,

hasta en Arabia procurar la fénix;

(Acte I, v. 804-811)

Dans Las bizarrías de Belisa, il est question d'une pierre précieuse nommée : "fénix de diamantes" (Acte II, v. 588). Le phénix revient très souvent comme comparant visant à exalter la beauté de la dama. Il est vrai que la fréquence d'utilisation du terme fénix peut donner l'impression d'une écriture quelque peu stéréotypée. Dans les vers suivants de El piadoso aragonés, on devine clairement qu'à travers les exclamations du personnage, il est question de ces clichés $^{19}$ :

\author{
CARLOS ;Qué de locos disparates! \\ Esto parece a la fénix, \\ que después de muerta nace \\ de sus cenizas al sol. \\ ¡Qué mentiras tan notables! \\ O como aquello del cisne, \\ que al morir con voz suave \\ canta, no habiendo en el mundo \\ quien haya visto que cante; \\ del pelicano se escribe \\ que el pecho a sus hijos abre, \\ necedad, pues mejor fuera \\ darles trigo, que no sangre;
}

17. Lactance dans Carmen de ave phøenice insiste également sur le caractère unique de l'oiseau: " unica semper avis ». Cf. Alain Goulon, "L'oiseau Phénix de Lactance et ses attaches à l'œuvre apologétique ", in Phénix : mythe(s) et signe(s), Actes du colloque international de Caen (12-14 octobre 2000), Berne, Berlin, Bruxelles, éd. Silvia Fabrizio-Costa, Peter Lang, 2001, p. 85-103.

18. Lope de Vega, Peribáñez, Madrid, éd. de Juan María Marín, Cátedra, «Letras Hispánicas », 2003.

19. Lope de Vega, El piadoso aragonés, Alicante, éd. de la Biblioteca Virtual Miguel de Cervantes, 2004, édition réalisée à partir de Veinte y una parte verdadera de las Comedias del Fenix de España Frei Lope Felix de Vega Carpio [...] En Madrid, por la viuda de Alonso Martin, a costa de Diego Logroño [...], 1635. 


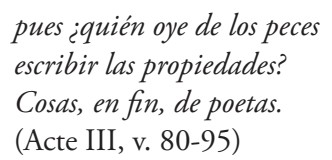

La voix du dramaturge affleure dans ce passage métathéâtral où il raille l'utilisation poétique du mythe du phénix. Peut-on avancer l'hypothèse selon laquelle Lope ne se contente pas de ce stéréotype ? En réalité, il semble que, même dans les utilisations les plus anodines, Lope renouvelle la caractérisation du phénix. Nous prenons pour preuve Amar, servir y esperar où le dramaturge invente un phénix qui renaît de la neige. Pour louer la blancheur des mains de Dorotea, Feliciano les compare à la neige ${ }^{20}$ :

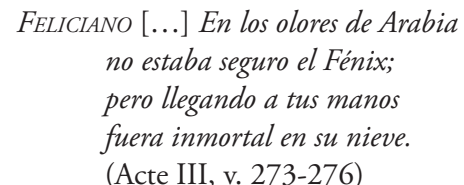

Dans la comedia lopesque, l'oiseau-phénix est sujet à diverses transformations poétiques. Il est fréquemment associé à l'amour dans de nombreuses pièces comme on peut le constater dans ces vers de El desprecio agradecido ${ }^{21}$ :

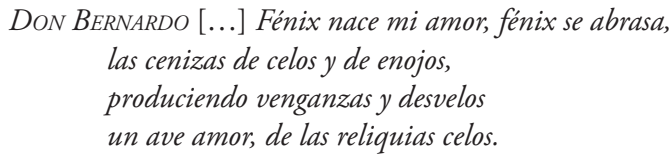

DON BERNARDO [...] Fénix nace mi amor, fénix se abrasa,

las cenizas de celos y de enojos,

produciendo venganzas y desvelos

un ave amor, de las reliquias celos.

(Acte II, v. 235-238)

Ces vers témoignent d'une innovation poétique du mythe. Dans la typologie du phénix, l'amour-phénix est l'un des types que Lope réinvestit dans son théâtre. Ici, le dramaturge crée l'amour-phénix à partir du ressort dramatique de cette comedia de capa y espada c'est-à-dire la jalousie (" celos »). Il s'agit en quelque sorte d'un phénix "a lo profano ", propre à la comedia de enredo.

Comme dans El peregrino en su patria, Médée apparait curieusement dans les vers faisant suite au fragment cité ci-dessus de Amar, servir y esperar ${ }^{22}$. L'évocation de la magicienne après le mythe du phénix est fréquente et il semble que Lope rassemble les mythes ovidiens en faisant converger leur symbolisme ${ }^{23}$.

20. Lope de Vega, Amar, servir y esperar, Alicante, éd. de la Biblioteca Virtual Miguel de Cervantes, 2005, édition réalisée à partir de Ventidos parte perfeta de las Comedias del Fenix de España Frey Lope Felix de Vega Carpio [...] En Madrid, por la viuda de Juan Gonçalez, a costa de Domingo de Palacio y Villegas y Pedro Verges, 1635.

21. Lope de Vega, El desprecio agradecido, Alicante, éd. de la Biblioteca Virtual Miguel de Cervantes, 2003, édition réalisée à partir de Parte veintecinco, perfeta y verdadera, de las comedias del Fenix de España Frey Lope Felix de Vega Carpio [...] Zaragoza, Por la viuda de Pedro Verges, a costa de Roberto Devport, 1647.

22. FELICIANO No importaran a Medea

dragones, ni toros fuertes,

porque sus manzanas de oro

trujera en sus ramos verdes. (Acte III, v. 277-280)

23. Rappelons que le mythe de Médée est développé dans le septième livre des Métamorphoses. 
La sorcière antique fait également partie de l'entourage textuel du terme fénix dans Angélica en el Catay ${ }^{24}$ :

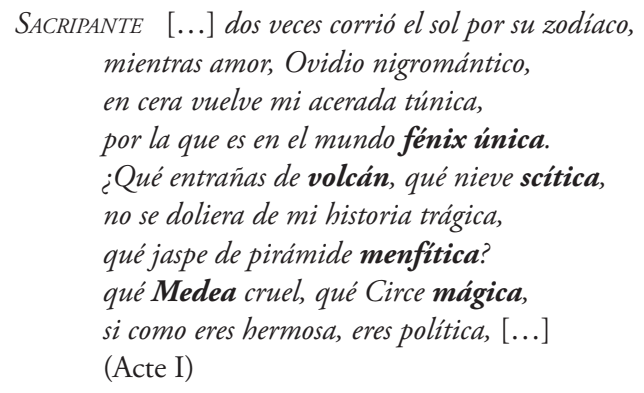

Ces vers, où riment des mots "esdrújulos", offrent une métamorphose particulière : la tunique d'acier se transforme en cire. Dans Angélica en el Catay, comme le titre de la comedia le laisse présumer, Lope s'inspire de Roland furieux de L'Arioste ${ }^{25}$. Sacripante, le roi de Circassie, s'est épris d'Angélica et la poursuit. Dans la pièce, il n'éprouve que des désirs charnels à son égard. Les vers qu'il prononce en aparté en sont l'illustration :

\section{SACRIPANTE Necia es la mujer, que fía en palabras de hombre que ama. (Acte I)}

Sacripante apparaît comme un personnage particulièrement démoniaque qui rappelle le don Juan tirsien. Face au dédain de la «dama», Sacripante prononce cette tirade où il exhale ses plaintes. Le premier vers « dos veces corrió el sol por su zodíaco " met l'accent sur un temps évoqué : les deux années durant lesquelles le personnage s'est entêté à poursuivre la "dama " en vain. Avant tout, ce passage s'apparente à une subjectivisation du temps évoqué par le personnage. J'entends par temps subjectif la temporalité perçue par le personnage durant cette période. Les deux années d'amour vain sont consacrées à un amourphénix puisque la dame est appelée "fénix única». Par ailleurs, il faut noter que Lope a souvent recours à l'astrologie comme une mesure du temps. Dans ces premiers vers, l'amour est personnifié : « Ovidio nigromántico». Le dramaturge convoque la figure d'Ovide, apposée à " amor ", qui devient agent de cette métamorphose cristallisée dans le verbe "vuelve ». Le maître d'œuvre de cette transformation magique n'est pas n'importe quel Ovide puisqu'il s'agit d'un Ovide nécromancien. Le terme "nigromántico " confère à la métamorphose un caractère occulte, proche de la magie noire. Comme le rappelle Covarrubias, la nécromancie était une pratique interdite par l'Église $e^{26}$. Cette méthode de

Pour plus d'informations sur le mythe de Médée dans le théâtre de Lope, cf. Juan Antonio Martínez Berbel, « "Puso el honor dragones de Medea”. Sobre ésta y otras Medeas en el teatro de Lope" ", Criticón, 87-88-89, 2003, p. 479-492.

24. Lope de Vega, Angélica en el Catay, Madrid, éd. Jesús Gómez, Paloma Cuenca, Turner, 1994. 25. L'Arioste, Roland furieux, Paris, préface d'Yves Bonnefoy, traduction de Francisque Reynard, Gallimard, " collection Folio classique », 2003.

26. "Nigromancia. Arte de adivinar invocando los muertos [...]. Esta arte y otras como chyromancia, hydromancia, geomancia, etc., están prohibidas por los sacros cánones, y últimamente 
divination par l'invocation des morts relève des sciences occultes et ce n'est pas un hasard si l'on retrouve par la suite l'évocation explicite des deux célèbres sorcières de l'Antiquité, Médée et Circé, et la référence à "la nieve scítica", la Scythie, région du Caucase connue pour ses sorcières et citée également par Ovide dans les Métamorphoses peu avant le passage sur le phénix ${ }^{27}$. De plus, les entrailles du volcan rappellent la figure de Vulcain, dieu du feu et des forges. Dans le terme "nigromántico", les notions antithétiques de mort (necro/nigro) et d'avenir (manteia/mántico) sont associées comme pour le phénix. Par ailleurs, il n'est pas impossible que Lope joue sur l'association des fricatives sourdes et sonores de "O-vi-dio / O-fi-dio » créant en quelque sorte, à travers la métamorphose des sonorités poétiques, un "Ovide ophidien ». La métamorphose, par liquéfaction, de l'armure de Sacripante en cire évoque, a contrario, le travail du forgeron et la technique de la cire perdue, ici liée au trait mortuaire de l'évocation. L'empreinte des Métamorphoses d'Ovide est encore visible à travers la "pirámide menfitica", dans la mesure où l'oiseau phénix, comme le précise Ovide, retourne en Égypte après sa renaissance, à Héliopolis, ville non loin de Memphis ${ }^{28}$. Par rapprochement, le terme «menfitico» rappelle aussi "mefitico", ce qui suggère que la pureté du mythe ovidien est contaminée par la magie noire. En filigrane, Lope laisse entendre qu'il ne s'agit plus d'un bûcher odorant mais d'une transformation dont l'exhalaison est fétide. C'est à une métamorphose de l'amour-phénix que nous assistons. Il ne s'agit pas d'un amour divin et pur mais d'un amour démoniaque lié aux penchants les plus vils, d'un amour sorcier. Dès lors, le phénix apparaît également comme l'oiseau de cette métamorphose occulte. L'expression poétique du phénix est bien retranscrite par le statut de " amor, Ovidio nigromántico ». L'amour est la cause de la métamorphose mais aussi son résultat. Dans ce passage, Lope établit une convergence des mythes. Au Moyen Âge, Ovide était considéré comme un nécromancien ${ }^{29}$. Le dramaturge rapproche Ovide et L'Arioste car le terme "nigromántico " fait probablement référence au Roland furieux où nécromanciens et magiciens sont convoqués à plusieurs reprises. Il est à noter qu'une des comédies de l'auteur italien s'intitule Il negromante ${ }^{30}$.

por el santo Concilio Tridentino. Nigromántico, el que usa desta superstición ". Sebastián de Covarrubias, Tesoro de la lengua castellana o española, Barcelone, Ad litteram, 3, Editorial Alta Fulla, 2003, p. 829.

27. On peut lire à ce sujet : Ovide, Les Métamorphoses, op. cit., p. 492 : "C'est ainsi encore que les femmes de Scythie, assure-t-on, à l'aide de sucs magiques qu'elles répandent sur leurs membres, opéreraient le même prodige. ". Pour plus d'informations sur les sorcières dans l'Antiquité, cf. Mickaël Martin, Sorcières et magiciennes dans le monde gréco-romain, en particulier "Métamorphose et métamorphoses ", p. 379-391, éd. manuscrit. com, 2004.

28. Ovide, Les Métamorphoses, op. cit., p. 133. L'éditeur précise en note au sujet d'Hypérion : "La ville d'Hypérion n'est autre qu'Héliopolis en Basse Égypte ".

29. Cf. Giovanni Pansa, Ovidio nel medioevo e nella tradizione popolare, Sulmona, éd. Ubaldo Caroselli, 1924, p. 33.

30. L'Arioste, Il negromante, Milan, Rizzoli Editore, 1962. 


\section{LE PHÉNIX : UNE STRUCTURE À USAGE DRAMATIQUE}

Les exemples présentés jusqu'ici ne concernent pas l'architecture temporelle de la pièce en termes de chronologie dramatique puisqu'il s'agit de motifs poétiques n'affectant pas le sens global de la structure temporelle de l'œuvre. Il convient de prendre en considération à présent l'autre versant de l'usage du mythe dans le théâtre de Lope.

On observe, dans de nombreuses pièces à matière historico-légendaire, des tirades contenant de longues déclinaisons de rois successifs. Il s'agit la plupart du temps d'un rappel des ancêtres d'un personnage illustre ou bien, en particulier dans les prophéties, de ses successeurs. Le phénix est parfois cité dans ces tirades apologétiques qui n'ont pas simplement une fonction idéologique visant à louer la monarchie. L'apparition du phénix peut paraitre stéréotypée mais nous pensons que sa présence ainsi que l'extension des limites du temps évoqué permettent une ouverture temporelle dans la pièce. Dans cette configuration, le mythe du phénix n'est pas développé et seul son nom apparaît. Nous trouvons un autre type d'allusion : un phénix apologétique. Ce cas de figure est présent dans le deuxième acte de Amar, servir y esperar:

\section{DIEGO ;O generosa ciudad! \\ del Fénix la eternidad \\ siglos pacificos vivas. \\ (Acte II, v. 502-504)}

L'évocation de l'éternité du phénix produit un effet d'élargissement des limites du temps évoqué dans le cadre temporel très bref de l'acte II où l'action s'étend sur deux jours successifs.

Il est certain que la temporalité du lignage est une préoccupation dans la poétique du temps de Lope. El piadoso veneciano ${ }^{31}$ montre à quel point l'application du mythe du phénix sert la poétique globale de l'œuvre. L'amour de Fulgencio dans El piadoso veneciano peut être comparé à celui de Sacripante de Angélica en el Catay car, à l'origine, la volonté de ces personnages est de séduire la "dama" par un moyen illicite. En effet, Fulgencio veut débaucher Lucinda, la femme de Sidonio. À la fin de l'acte I, Fulgencio est tué par le mari jaloux. C'est six ans plus tard, au début du troisième acte, qu'Otavio, fils de Fulgencio, souhaite venger la mort de son père et se rend chez Lucinda pour lui demander des comptes. On retrouve d'ailleurs Circé impliquée dans le processus des métamorphoses que les insultes font subir à la traitresse :

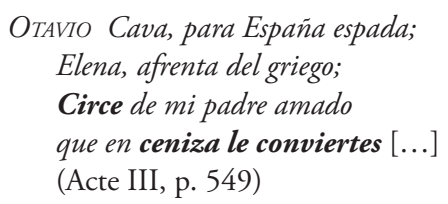

31. Lope de Vega, El piadoso veneciano, Obras completas, Lope de Vega, Comedias XV, Madrid, éd. Jesús Gómez, Paloma Cuenca, Turner, «Biblioteca Castro », 1998, p. 487-573. 
Dans la dernière jornada, Otavio est devenu un homme et il s'éprend d'Elisa, la fille du couple Sidonio / Lucinda. La jeune femme est également amoureuse d'Otavio. Le mythe du phénix est mis à contribution dans les deux derniers tercets du sonnet où Elisa déclare son amour pour le galant qui souhaitait s'en prendre à sa mère :

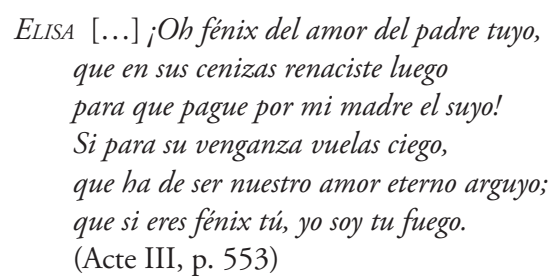

L'oiseau phénix a été introduit quelques vers auparavant par le terme «ceniza» et la métamorphose agie par Circé. L'image de l'amour-phénix est liée, dans le cas présent, à la succession des générations et des amours et à la temporalité de la pièce. La réconciliation des deux familles ennemies est scellée par l'union des enfants, ce qui permet la perpétuation du lignage. Lope réécrit à nouveau une métamorphose du phénix à usage dramatique et poétique cette fois. Ce cas de figure est également présent dans El bastardo Mudarra où l'héritier de Gonzalo Bustos promet de venger les sept infants de Lara ${ }^{32}$ :

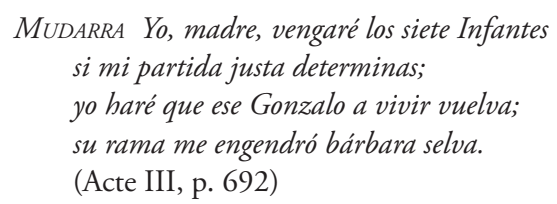

Mudarra veut transcender la mort tragique de Gonzalo en tuant Ruy Velázquez, personnage à l'origine de la mort des infants. Il permet de redonner à Gonzalo Bustos une succession et s'inscrit dans la lignée des Lara. Cette comedia met en scène le motif de la vengeance différée d'une génération à une autre. Mudarra, vengeur providentiel, est l'héritier permettant de perpétuer le lignage de Gonzalo Bustos. Sa bâtardise relative se dissout progressivement, le mariage avec sa nièce Clara, la fille de son demi-frère Gonzalo González, permettant le rétablissement de la lignée familiale. Avant de rencontrer doña Clara, Lope, un écuyer, présente la "dama " à Mudarra en l'assimilant au phénix :

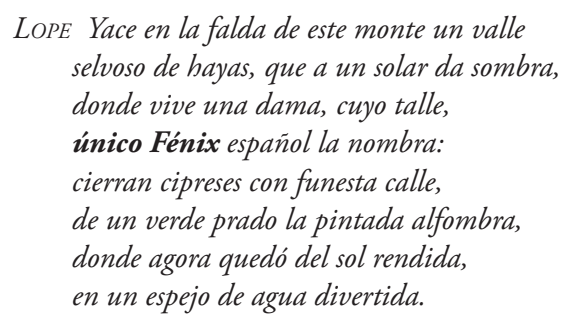

32. Lope de Vega, El bastardo Mudarra, Obras escogidas, tomo III, teatro II, Madrid, éd. Federico Carlos Sainz de Robles, Aguilar, 1990, p. 667-702. 


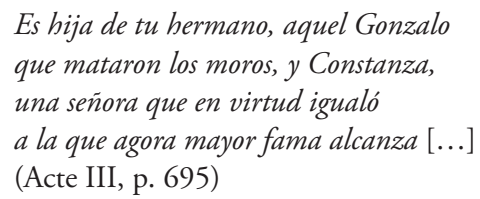

C'est par l'intermédiaire de Clara, unique phénix et phénix unique, que Mudarra permettra la perpétuation de son lignage.

Une autre comedia historico-légendaire met à contribution le mythe du phénix au sein de l'architecture temporelle. El postrer godo de España ${ }^{33}$ représente l'histoire bien connue de don Rodrigo. Les deux premiers actes mettent en scène la tragédie du dernier roi wisigoth. À la fin de l'acte II, Rodrigo meurt lors de la célèbre bataille de Guadalete, qui eut lieu en 711. La dernière jornada suit l'ascension de Pelayo, qui résiste aux Arabes dans les Asturies à l'occasion du non moins fameux affrontement de Covadonga, en 722 . La pièce comporte deux époques différentes : le règne de Rodrigo, qui ne dura qu'un an (710711), et l'action menée par Pelayo se déroulant une dizaine d'années plus tard. Au troisième acte, Pelayo est considéré comme le "Fénix de los muertos godos " (v. 2727). Vers la fin de la comedia, Pelayo se proclame phénix de l'Espagne dans un sonnet :

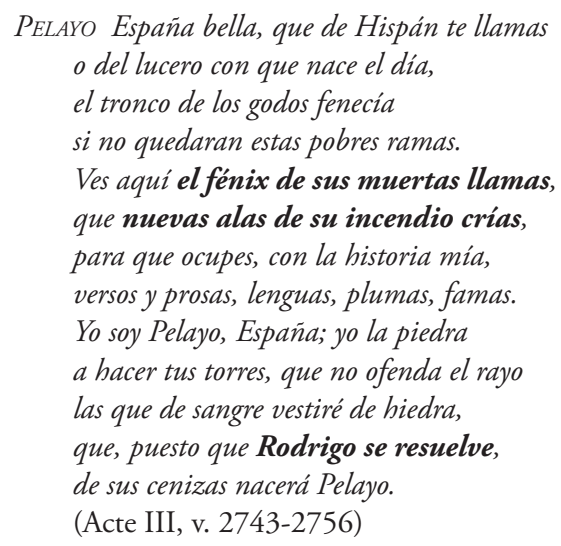

La mort tragique de Rodrigo au deuxième acte provoque une rupture temporelle dans la comedia qui se traduit par l'imprécision de la durée de l'action dans le deuxième inter-acte. La reconquête du territoire s'apparente à une entreprise de raccommodage du temps perdu. En faisant renaître Pelayo des cendres de Rodrigo, le dramaturge subvertit la perte tragique du dernier roi wisigoth. La défaite chrétienne des deux premiers actes est transmuée en victoire par Pelayo, dont la vibrante invocation à l'Espagne donne un sens mythique à l'histoire, un héros nouveau naissant des cendres du roi vaincu. Le dramaturge procède de la même façon que dans El piadoso veneciano ou $E l$ bastardo Mudarra puisque la tentative avortée du père ou de l'aïeul est remise

33. Lope de Vega, El postrer godo de España, éd. Jorge García López, Comedias de Lope de Vega, parte VIII, vol. II, coord. Rafael Ramos, Lérida, Milenio, Universitat Autònoma de Barcelona, 2009. 
en question et rénovée par la poétique d'un temps-phénix. Dans El postrer godo, la tragédie collective des deux premiers actes est transcendée, ce qui donne naissance à un nouveau cycle épico-historique.

Le mythe du phénix, intrinsèquement lié à la notion de renaissance, a été abondamment utilisé comme le symbole chrétien de la résurrection ${ }^{34}$. Il est curieux de constater que, dans les citations du corpus hagiographique, le mythe de l'oiseau merveilleux n'est pas aussi développé que dans les comedias profanes. Il existe néanmoins un phénix " a lo divino ", ayant des caractéristiques propres. Nous citerons des exemples extraits de deux pièces : El niño inocente de la Guardia et El divino africano.

L'empreinte christique de El niño inocente de la Guardia est indéniable car la comedia représente le martyre d'un enfant chrétien. Juanico est capturé par des juifs au deuxième acte pendant l'Assomption et il sera crucifié huit mois plus tard, durant la Pâque juive. Dans les derniers vers de la pièce, Juanico est comparé au phénix :

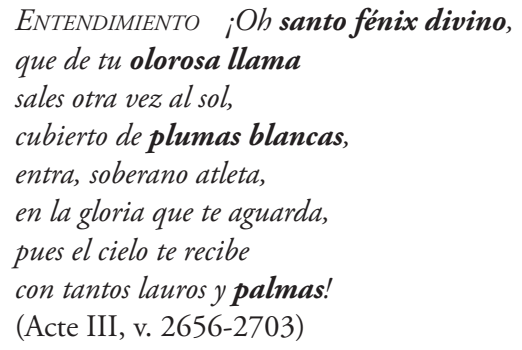

Le mythe ovidien est christianisé par Lope et les éléments entrant en jeu dans la métamorphose sont bien différents. Il ne s'agit plus de nécromancie ou de sorcellerie et les images visuelles de fusion du métal ou de combustion laissent la place à la lumière, à la blancheur immaculée et à la flamme odorante, également présente chez Ovide. Le mythe du phénix entre dans le cadre d'un temps divin, supra-terrestre lié à l'éternité, qui transparaît à travers le palmier (également cité par Ovide) et le paradis ("gloria»). Comme dans El peregrino en su patria, Lope fait allusion à un oiseau blanc, une colombe renaissante qui rappelle l'Esprit-Saint.

Dans El divino africano, mettant en scène la vie de saint Augustin, nous retrouvons l'image chrétienne de la résurrection. Mónica, la mère d'Augustin, est à la recherche de son fils ${ }^{35}$ :

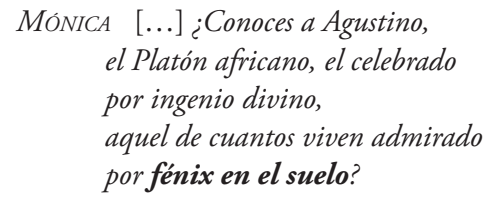

34. Cf. Paul-Augustin Deproost, in "L'oiseau entre ciel et terre ", Paris, Association Kubaba, L'Harmattan, 2005, p. 113-138.

35. Lope de Vega, El divino africano, Obras escogidas, tomo III, teatro II, Madrid, éd. Federico Carlos Sainz de Robles, Aguilar, 1990, p. 206-237. 


\section{¡Ay, si lo fuese en renacer al cielo!}

(Acte I, p. 213)

Ces deux vers résument bien la temporalité particulière de la comedia hagiographique. Le saint participe de deux temporalités : le temps humain et le temps divin. Le phénix cristallise bien la réunion de ces deux plans temporels. L'oiseau phénix est un trait d'union entre le temps terrestre (" en el suelo») et l'au-delà (" al cielo»). Dans l'hagiographie, le mythe du phénix est épuré. On ne retrouve plus les descriptions "baroques » des métamorphoses. On se contente de le nommer. Considérer le futur saint comme un phénix, c'est reconnaître dès lors son éternité. Les vers suivants appuient cette observation :

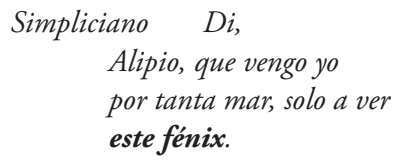

(Acte III, p. 233)

Vers la fin de la pièce, la mort et l'au-delà sont rapprochés :

ALIPIO Señor,
en este momento goza
de la eternidad de Dios.
ULDERICO ¿Es muerto?
ALIPIO Otra vida cobra.

(Acte III, p. 237)

La proximité des termes "muerto " et "otra " et le phénomène de synalèphe, réalisé du moins à la lecture du vers, produit une fusion entre la mort et l'audelà ; "muertotra vida " illustre cette métamorphose divine qui n'est autre qu'une résurrection.

Le rituel palingénésique antique est réadapté par Lope de Vega. Dans les comedias profanes, l'amour-phénix est souvent lié à la magie noire quand le personnage est animé de mauvaises intentions. Lope de Vega crée un Ovide nécromancien et convoque plusieurs figures mythologiques dont les sorcières, établissant ainsi une poétique du syncrétisme mythique. C'est le caractère magique du mythe qui est mis en valeur à l'aide de descriptions particulièrement évocatrices du sous-sol et des entrailles de la terre. La métamorphose de l'oiseau phénix est l'expression du temps subjectif caractérisant - on l'a vu - le temps évoqué dans Angélica en el Catay. L'amour-phénix n’est pas exclusivement un motif ; il peut être une structure à usage dramatique et il est également associé - El piadoso veneciano en est la preuve - au temps du lignage et des générations. Lope de Vega utilise l'oiseau phénix dans une poétique de la perpétuation et le rajeunissement cycliques de l'ancêtre. Nous pourrions même parler de tempsphénix pour qualifier cette temporalité visant l'éternité.

Une certaine verticalité est remarquable dans le traitement du mythe du phénix par Lope. Les tréfonds magiques et occultes où se reproduit l'oiseau du feu passionnel et, par contraste, les cieux accueillant l'envol de la colombe renaissante. Dans la comedia de santos, Lope a recours à des images éthérées de la métamorphose. Seuls les contrastes semblent primer. L'oiseau fabuleux 
ovidien est christianisé au service d'une jonction des deux plans temporels de l'hagiographie : le temps humain (la mort) et le temps divin (l'au-delà). Les différents exemples ont montré à quel point Lope de Vega, poète, joue avec la métamorphose et parvient à transformer le langage (pensons aux allitérations du Peregrino ou au jeu des sonorités dans Angélica en el Catay). Il serait souhaitable d'étudier les liens du mythe du phénix avec d'autres éléments comme le « mortvivant ", analysé par Maria Aranda dans un récent article ${ }^{36}$, ou le "puer senilis ». Il resterait également à étudier le phénix comme personnage scénique car les noms Fénix ou Fenisa sont souvent utilisés dans certaines pièces ${ }^{37}$.

Il est également une caractéristique du phénix qui a trait à l'hagiographie : son hermaphroditisme ${ }^{38}$. Cet oiseau merveilleux, chaste et abstinent qui ne se nourrit que du suc de l'amome, est un type parfait de sainteté39. L'hermaphroditisme du phénix est aussi exploité par Lope, ce qui lui permet d'attribuer indifféremment le nom du phénix à des dames et à des galants.

Lope de Vega recrée dans son théâtre un phénix protéiforme, un "phénix ophidien " des profondeurs de Vulcain ou un phénix de la gloire céleste, un phénix permettant le rétablissement du lignage et subvertissant la perte tragique, un phénix intégré dans la structure temporelle de la comedia et vecteur de métamorphoses du temps.

36. Maria Aranda, "De Circé à Ulysse : le naufragé "gothique" de Lope de Vega ", Bulletin Hispanique, tome 112, n 1 , coordonné par Federico Bravo, Presses Universitaires de Bordeaux, juin 2010, p. 61-73.

37. C'est le cas de Servir a buenos ou de El poder en el discreto par exemple.

38. Bachelard définit le phénix en ces termes : "Le Phénix est une somme de valeurs poétiques, jeu de multiples correspondances : feu, baume, chant, vie, naissance, mort. Il est nid et espace infini. Il a les deux chaleurs, du nid et du soleil. Chaleur du chant, chaleur du baume, tout converge pour enflammer l'oiseau : feux masculins des chants qui réveillent, chaleur féminine berçante de l'aromate qui endort, voilà encore une transposition de plus en plus fine, donc de plus en plus vraie, de l'hermaphroditisme de la grande image ". Gaston Bachelard, op. cit., p. 102.

39. Paul-Augustin Deproost dans "Les métamorphoses du phénix dans le christianisme ancien " précise au sujet de ce caractère asexué du phénix : "L'unicité du phénix lui mérite, enfin, une dernière qualité hautement valorisée dans le christianisme primitif : la virginité. "Mâle ou femelle, ou bien ni l'un ni l'autre ou bien l'un et l'autre", comme le dit Lactance, l'oiseau unique est un être tout à la fois bisexué, qui rappelle l'androgynie de l'homme primitif "à l'image et à la ressemblance de Dieu", dans les exégèses juives du premier récit de la création, et asexué, qui profile l'idéal paulinien de la vie dans le Christ et l'état des ressuscités, car, selon Jésus lui-même, "à la résurrection, on ne prend ni femme ni mari, mais on est comme des anges dans le ciel" ". Paul-Augustin Deproost, in «L'oiseau entre ciel et terre », L'Harmattan, 2005, p. 113-138. 
Liste des comedias du corpus

Amar, servir y esperar

Angélica en el Catay

Argel fingido

Contra valor no hay desdicha

Don Juan de Castro (Primera parte)

El bastardo Mudarra

El caballero de Olmedo

El caballero del sacramento

El capellán de la Virgen

El cardenal de Belén

El casamiento en la muerte

El castigo sin venganza

El desprecio agradecido

El divino africano

El mayor imposible

El niño inocente de la Guardia

El nuevo mundo

El piadoso aragonés

El piadoso veneciano

El postrer godo de España

El remedio en la desdicha

El sembrar en buena tierra

El testimonio vengado

La amistad pagada

La bella Aurora
La Burgalesa de Lerma

La comedia de Bamba

La dama boba

La desdichada Estefanía

La hermosa Ester

La imperial de Otón

La noche de san Juan

La noche toledana

La ocasión perdida

La octava maravilla

La piedad ejecutada

La resistencia honrada

Las bizarrías de Belisa

Las paces de los reyes

Lo fingido verdadero

Los comendadores de Córdoba

Los melindres de Belisa

Los palacios de Galiana

Los porceles de Murcia

Los prados de León

Los Ramírez de Arellano

Lucinda perseguida

Peribánéez y el comendador de Ocaña

Roma abrasada

Servir a buenos 
